EPJ Web of Conferences 110,01001 (2016)

DOI: $10.1051 /$ epjconf/201611001001

(C) Owned by the authors, published by EDP Sciences, 2016

\title{
DETERMINATION OF MAINTAINING TIME OF TEMPERATURE TRACES OF AEROSOL DROPLET WATER FLOWS DURING MOTION IN A FLAME
}

\author{
D.V. Antonov ${ }^{1, a}$, I.S. Voitkov ${ }^{1}$, P.A.Strizhak ${ }^{1}$ \\ ${ }^{1}$ National Research Tomsk Polytechnic University, 634050 Tomsk, Russian
}

\begin{abstract}
To develop fire fighting technologies, the temperatures of combustible products were measured after passing an aerosol droplet flow of water through the flames (with monitored temperatures). It was applied the aerosol flows with droplets of sizes less than $100 \mu \mathrm{m}, 100-200 \mu \mathrm{m}$, and 200-300 $\mu \mathrm{m}$. Investigations were conducted at a temperature of combustible products from $500 \mathrm{~K}$ to $900 \mathrm{~K}$. Temperatures of gases in droplet flow traces and maintaining times of relatively low temperatures in these areas (it can be considered as temperature trace) were defined. It was obtained the satisfactory agreement of experimental results and numerical simulation data.
\end{abstract}

\section{Introduction}

Over the years, the problem of fire protection is an urgent one due to the large material losses (scale consequences of fires, particularly, forest ones). Thus, the main objective of various developments and researches is the evolution of modern tools and methods of fire fighting. Currently, there are three main mechanisms of fire fighting [1,2]: cooling the heat source due to the implementation of water droplet evaporation (the phase transformation of first and second type occurs at the same time); insulation of heat source due to covering its surface with a layer of gas forming when evaporation of fine flow of water droplets (thus, the ignition process is practically not supported); simultaneous cooling and insulation of the heat source. By using these methods of fire fighting, one focuses on such parameters as mutual bracing of atomizers, spray speed, water discharge, dispersability of droplets in a flow [3-5]. The influence of temperature and concentration traces of droplet water flows during motion in a flame on characteristics of fire extinguishing has not been studied. All this is due to an insufficiency of valid experimental data for corresponding traces of droplet water flows.

In recent years, only theoretical investigations [5-7] of temperature traces of aerosol droplet flows of water during motion in a flame were conducted. The insufficiency of experimental data is due to difficulty in experimentations according an application necessity of quick-response thermocouples which have a number of drawbacks [8]: high cost and sensitivity to deformations.

The aim of the present work is to determine experimentally the maintaining times of temperature traces of aerosol droplet water flows during motion in a flame.

\section{Experimental setup and procedure}

In the performance of an experiment we used the experimental setup. Its scheme is presented in Fig. 1.

\footnotetext{
${ }^{\text {a }}$ Corresponding author: 1aadsdfn@gmail.com
} 


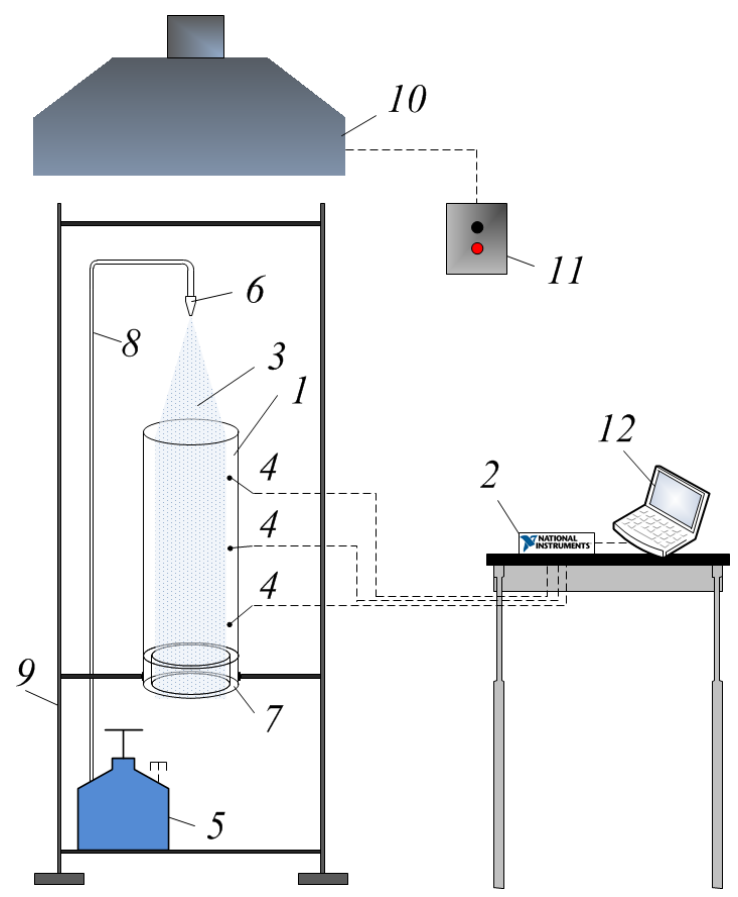

Figure 1. Scheme of experimental setup: 1 - cylinder of heat-resistant transparent material; 2 - specific device National Instruments; 3 - droplet flow of water; 4 - thermocouples; 5 - vessel with water; 6 - atomizer; 7 - hollow cylinder with a combustible liquid in its cavity; 8 - channel of water supply; 9 - aluminum rack; 10 - exhaust ventilation; 11 - switch on/off remote control of exhaust ventilation; 12 - personal computer.

The setup was used to record the maintaining times of high-temperature traces of aerosol droplet water flows (Fig. 2) in a cylindrical channel 1 , its height was $h \approx 1 \mathrm{~m}$. At each time point, the temperature recorded by specific complex National Instruments 2. The gas temperature $T_{\mathrm{g}}$ in a flame zone varied from $500 \mathrm{~K}$ to $900 \mathrm{~K}$.

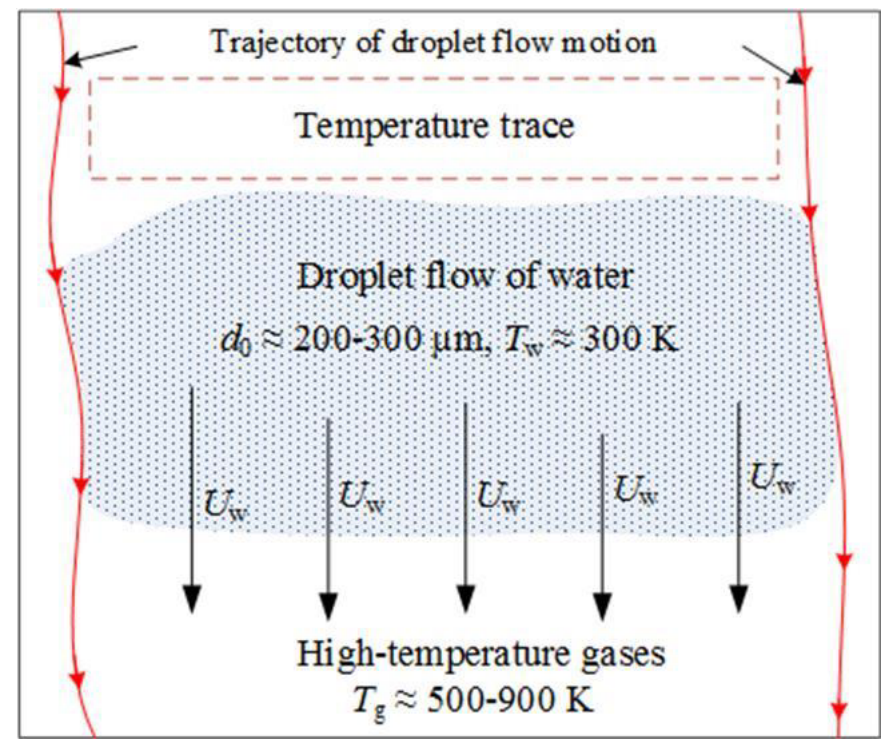

a 

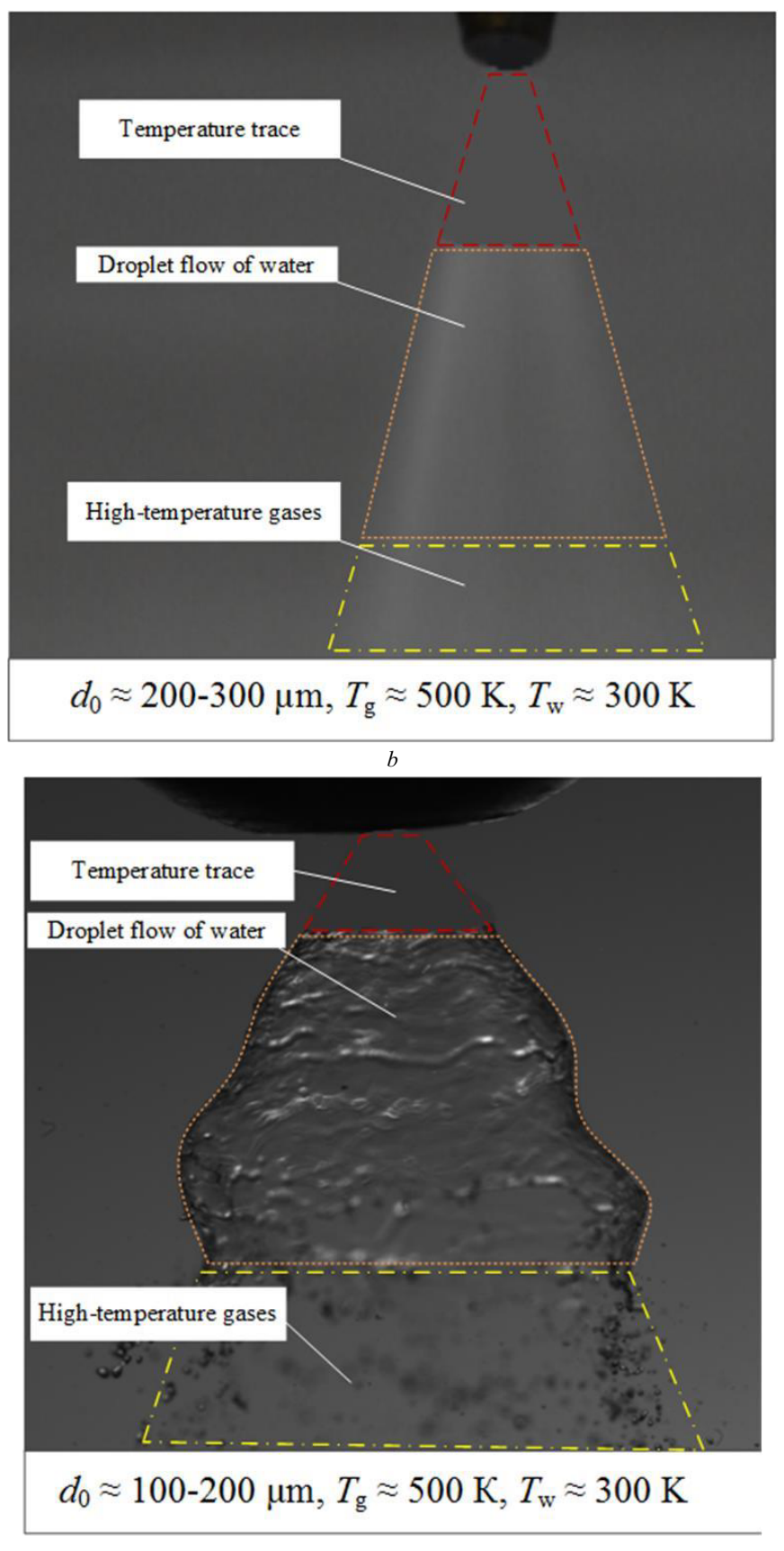

Figure 2. Temperature trace of droplet flow of water ( $a$ - scheme, $b$ - video frame with water fog, $c$ - video frame with water jet).

Flames of kerosene were formed in cylindrical channels (Fig. 3). We used channels from quartz, gland and stainless steel to determine the maintaining times of temperature traces of aerosol droplet water flows during motion in a flame. 


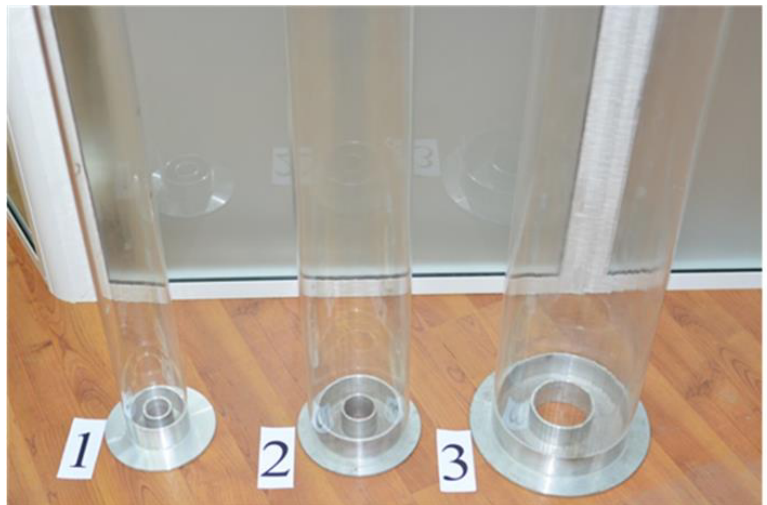

$a$

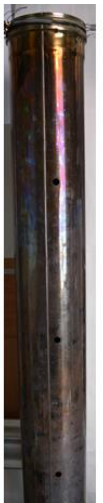

$b$

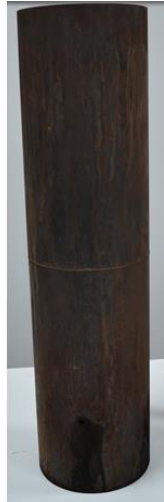

$c$

Figure 3. Picture of different cylindrical channels: $a$ - from quartz glass, the height is $h \approx 1 \mathrm{~m}: 1-D \approx 100 \mathrm{~mm}, 2-D \approx 150 \mathrm{~mm}, 3-D \approx 200$ $\mathrm{mm} ; b$-from stainless steel; $c$ - from the gland.

To determine the maintaining times of temperature traces of aerosol droplet water flows in a cylindrical channel 1 , we developed specialized techniques. Each series of experiments involves the following steps:

- internal cavity 7 of the channel 1 was filled with combustible liquid - kerosene in an amount of $300 \mathrm{ml}$, and its ignition was initiated;

- at the initial stage of kerosene combustion, the high-temperature gas medium filled the whole internal cavity of cylindrical channel 1. After establishing the required temperatures (about $900 \mathrm{~K}$ ), the droplet flow of water 3 in the form of individual pulses (a period of one impulse was 1-2 s, delay time between two pulses was $30 \mathrm{~s}$ ) of water was supplied to a flame zone with the height of $30 \mathrm{sm}$ from cylindrical channel 1 outlet (droplet flow of water covered the entire area of the cylindrical channel 1 );

- temperature of kerosene flames at each time point was measured with three chromel-alumel thermocouples 4 (range of temperature measurement from $223 \mathrm{~K}$ to $1473 \mathrm{~K}$, time of heat inertia 1-2 s). The temperature measurement error was \pm 3 $\mathrm{K}$;

- 15-20 series of measurements were carried out under identical initial conditions (spraying parameters);

- processing of the results was performed by the specialized software National Instruments.

To estimate motion velocity of high-temperature gas flow, we applied PIV method based on recording the instant velocity distributions of "tracers" in the flow of gases between the laser flashes. The "tracer" particles (we used titanium dioxide nanopowder) were injected in a gas flow in a top part of cylindrical channel 1 . Combustible product speeds $U_{\mathrm{g}}$ were about $1.5 \mathrm{~m} / \mathrm{s}$. The maximum systematic errors of measurement $U_{\mathrm{g}}$ were $0.01 \mathrm{~m} / \mathrm{s}$.

During the experiments, the spraying parameters were regulated. We performed the pulsed water supply (a period of one impulse was 1-2 s, delay time between two pulses was $30 \mathrm{~s}$ ). Sizes of aerosol water flow droplets were varied (fine spraying is water droplets of sizes (diameter) less than $100 \mu \mathrm{m}$; medium spraying is water droplets of sizes (diameter) 100-200 $\mu \mathrm{m}$; large spraying is water droplets of sizes (diameter) $200-300 \mu \mathrm{m}$ ).

To measure the dimension of droplet flow of water when large, medium and fine spraying, the SP method was applied. This method based on recording the shadow image of an object, the refraction coefficient of which is different to the environment. The diffused screen with a light guide was used to obtain the shadow image of moving droplets, later, in the processing of which their sizes and trajectories were determined. We applied fiber-optic light guide which was connected with diffused screen. Behind the screen was a diffuser that provided the required characteristics of the shadow illumination (radiation power and the opening angle of the laser light). Cross-correlation camera with pre-installed light filter recorded shadow images of water droplets. The postprocessing of images by using a number of filters and algorithms allowed determining the droplet size. Initial sizes (diameters) of droplets in an aerosol water flow varied within the range from $0.05 \mathrm{~mm}$ to $0.3 \mathrm{~mm}$ and they were: for large spraying $-200-300$ $\mu \mathrm{m}$, for medium one $-100-200 \mu \mathrm{m}$, for fine spraying - less than $100 \mu \mathrm{m}$. Relative volume density $\gamma_{\mathrm{m}}$ was maintained within the range $0.001-0.0012 \mathrm{~m}^{3}$ of liquid droplets in $1 \mathrm{~m}^{3}$ of gas. The maximum random errors in determining the parameters under consideration were $d_{\mathrm{m}}-2.1 \%, U_{\mathrm{g}}-3.4 \%$.

\section{Results and discussion}

Fig. 4 illustrates changes in temperature at different heights $(1-h \approx 0.12 \mathrm{~m}, 2-h \approx 0.34 \mathrm{~m})$ for medium spraying (initial diameter of droplets is $100-200 \mu \mathrm{m})$. It is clearly seen that temperature variations at direct heights are inconsiderable. The gas temperature is $T_{\mathrm{g}} \approx 500 \mathrm{~K}$ at a height of $h \approx 0.12 \mathrm{~m}$, and it achieves $T_{\mathrm{g}} \approx 150 \mathrm{~K}$ at a height $h \approx 0.34 \mathrm{~m}$. In a vicinity of hot construction surface, the gas temperature achieves $T_{\mathrm{g}} \approx 900 \mathrm{~K}$. While moving through the channel, temperature of combustible products is decreased. 


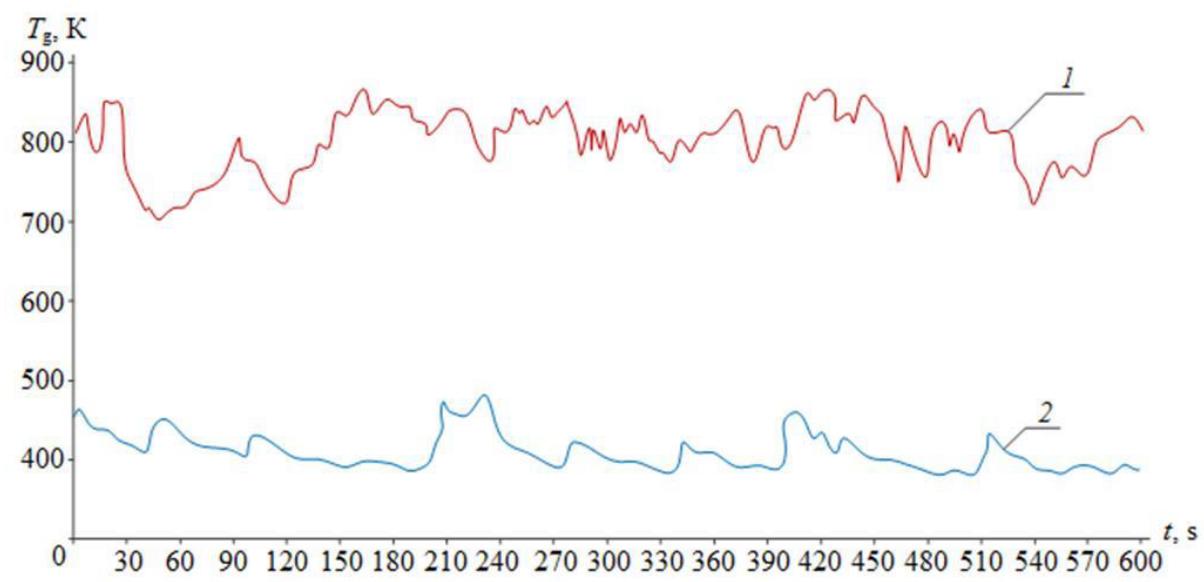

Figure 4. Temperature variations at different heights for medium spraying (initial sizes (diameters) of droplets are 100-200 $\mu \mathrm{m}$ ): $1-$ at $h \approx 0.12$ $\mathrm{m} ; 2-$ at $h \approx 0.34 \mathrm{~m}$.

Fig. 5 shows maintaining times $\tau$ of temperature trace from experiments of various types of spraying $(1-$ large spraying; 2 - fine spraying; 3 - medium spraying) and theoretical investigations by the model [4] ( 4 - for water array of dimension $0.1 \mathrm{~m} ; 5$ - for a single droplet of size $3 \mathrm{~mm}$ ). In Fig. 5 it is visible that due to the growth of temperature, maintaining times of temperature trace increase and they vary from $15 \mathrm{~s}$ to $20 \mathrm{~s}$. It is linked to the higher difference between the temperature of droplet trace and temperature of gases $\left(\Delta T=T_{\mathrm{g}}-T_{\mathrm{w}}\right)$.

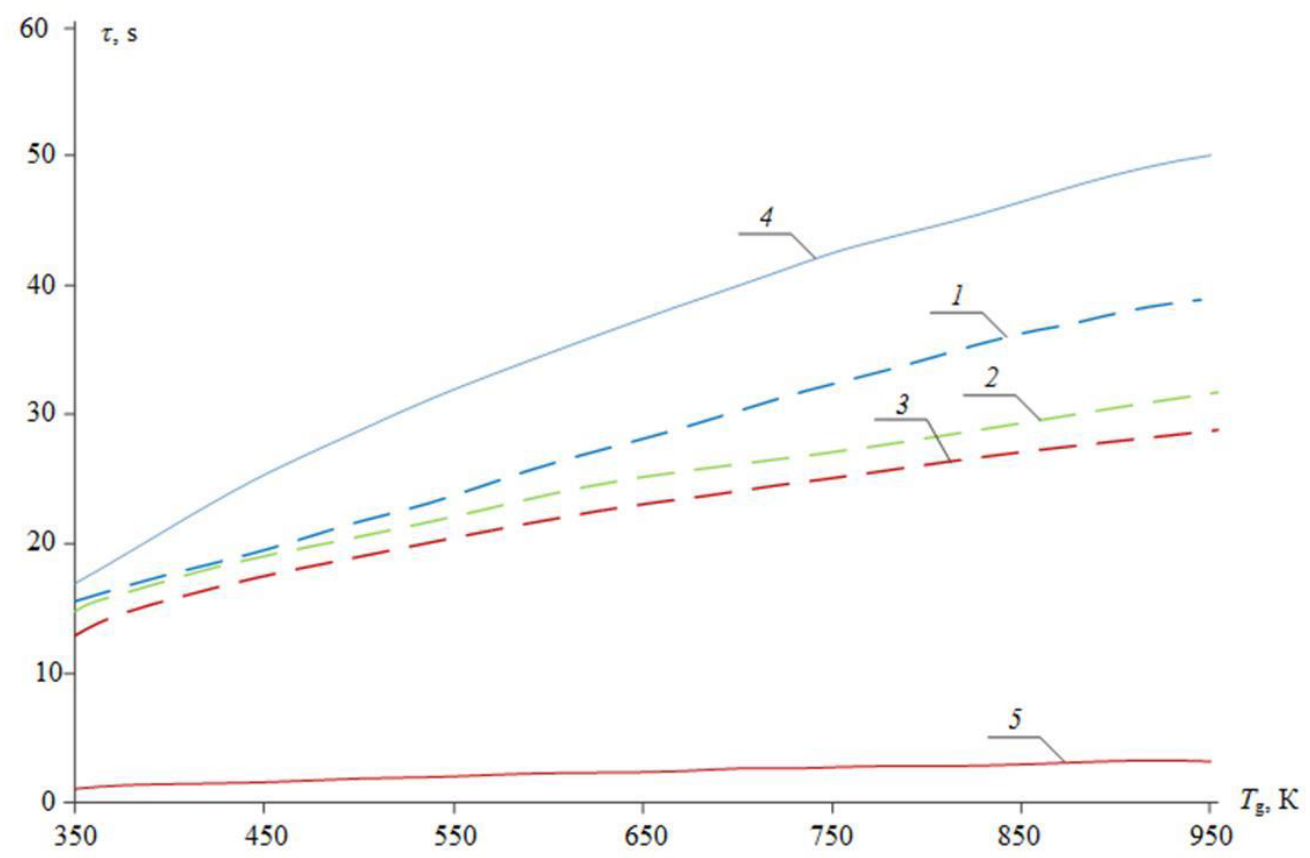

Figure 5. Maintaining times of temperature trace: $a$ - form experiments of various types of spraying: 1 - large spraying; $2-$ fine spraying; $3-$ medium spraying; $b$ - theoretical investigations [4]: 4 - for water array of dimension $0.1 \mathrm{~m} ; 5$ - for a single droplet of size $3 \mathrm{~mm}$.

From the conducted experiments, the variation ranges of maintaining times of temperature traces of aerosol droplet flows during motion in a flame were defined $\left(\tau_{\min } \approx 14 \mathrm{~s} ; \tau_{\max } \approx 30 \mathrm{~s}\right)$.

As a manner of experimentation experience, the differences for various materials of cylindrical channels were determined: quartz glass, stainless steel, gland. We established that the less heat is spending for heating the gland cylindrical channel. This is due to the different specific thermal capacity of materials of cylindrical channels. The specific thermal capacity of gland is $c=444$ $\mathrm{J} / \mathrm{kg} \cdot \mathrm{K}$, of stainless steel $-c=500 \mathrm{~J} / \mathrm{kg} \cdot \mathrm{K}$, of quartz glass $-c=1052 \mathrm{~J} / \mathrm{kg} \cdot \mathrm{K}$. As a consequence, conditions of temperature variation in a trace of aerosol flows differed sufficiently significantly. 


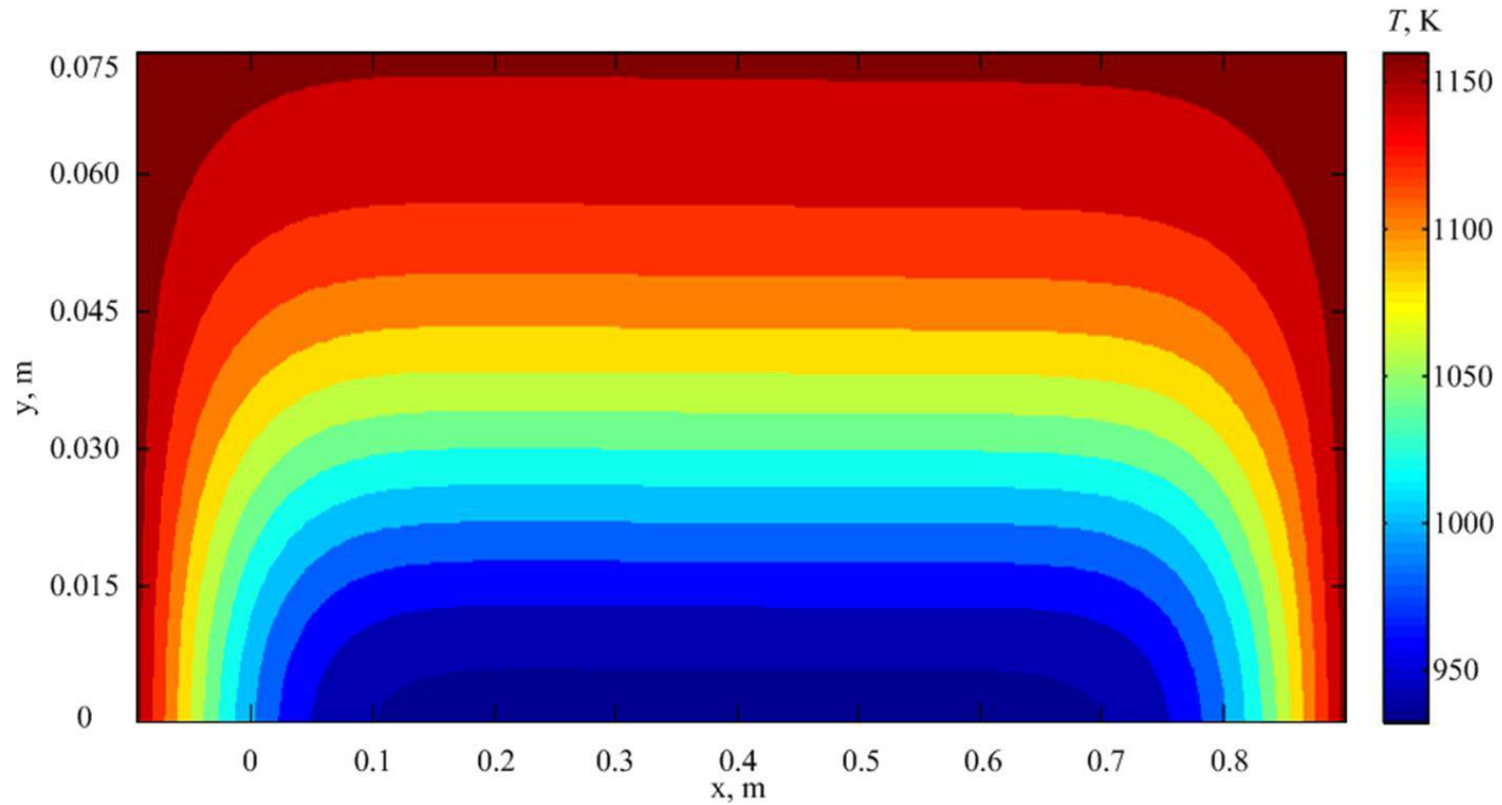

Figure 6. Temperature field (at the time moment of $3 \mathrm{~s}$ ) in a trace of water array of dimension (diameter) about $0.1 \mathrm{~m}$ obtained by the model [4].

Theoretical researches illustrated the satisfactory fit to the experimental investigations conducted in a laboratory setting (Fig. 5). Maintaining times $\tau$ of temperature trace at various gas temperatures $T_{\mathrm{g}}$ resulting from experimental investigations (we applied atomized droplet flow of water, the droplet sizes in which were varied within the range 50-300 $\mu \mathrm{m}$ ) order between dependences obtained from numerical investigations (thus, in one instance we considered a single water droplet of size $3 \mathrm{~mm}$, in another one water array of dimension $0.1 \mathrm{~m}$ ). This is due to that fact that a number of water droplets is significantly greater in the experiment than in numerical investigations, and these droplets are not monolithic, so they evaporate and form a whole cloud. Nevertheless, the obtained satisfactory correlation of theoretical and experimental times $\tau$ shows the application possibility of a group of models [4] to predict temperature in a trace of droplets, aerosol flows and large arrays during their motion through high-temperature gases.

\section{Conclusion}

The experimental investigations in determination of maintaining times of temperature traces of aerosol droplet water flows during motion in a flame (gas temperature was $T_{\mathrm{g}} \approx 500-900 \mathrm{~K}$ ) were conducted. Maximums and minimums of maintaining times of temperature trace were determined $\left(\tau_{\min } \approx 14 \mathrm{~s} ; \tau_{\max } \approx 30 \mathrm{~s}\right)$. The variation features of maintaining times of temperature trace with increasing of temperature were defined. The satisfactory correlation of theoretical and experimental investigations was demonstrated.

This work was supported by the Russian Science Foundation (project No. 14-39-00003).

\section{References}

1. S. K. Aggarwal, F. Peng, J., Eng. for Gas Turbines and Power. 117, 453 (1995)

2. A. Abbud-Madrid, D. Watson, J.T. McKinnon., Suppression and Detection Research and Applications Conference, (2007)

3. Y.V. Lyulin, O.A. Kabov., Appl. Phys. Lett. 39, 88 (2013)

4. P.A. Strizhak., J. Eng. Phys. and Thermophys. 86, 895 (2013)

5. S.S. Sazhin, A.E. Elwardany, P.A. Krutitskii, V. Depredurand, G. Castanet, F. Lemoine, E.M. Sazhina, M.R. Heikal., Int. J. Therm. Sci. 50, 1164 (2011)

6. J. E. Sprittles, Y. D. Shikhmurzaev., Phys. Fluids. 24 (2012)

7. R.V. Maltsev, A.K. Rebrov., J. Appl. Mech. and Tech. Phys. 48, 142 (2007)

8. H. Shenk., Mir Publisher, (1972) 